\title{
NORBERTO BOBBIO, PROFESOR
}

\author{
Michelangelo Bovero \\ Universidad de Turín
}

\begin{abstract}
RESUMEN. En este testimonio, el profesor Michelangelo Bovero recuerda la faceta de Norberto Bobbio como profesor. Partiendo por lo que fue su última clase, el autor da cuenta, por ejemplo, de la trayectoria de Bobbio en la docencia, el contenido y desarrollo de sus cursos, su metodología de enseñanza, su transición de la cátedra de Filosofía jurídica a la de Filosofía política, o su particular admiración y respeto por los clásicos. Así, mediante anécdotas, palabras, gestos y detalles de la personalidad del filósofo, el autor nos presenta tanto su visión de la faceta docente y humana de Norberto Bobbio como una despedida de su maestro.
\end{abstract}

ABSTRACT. In this account, professor Michelangelo Bovero remembers Norberto Bobbio in his role as a teacher. Taking his last lecture as a starting point, the author tells us, for example, about Bobbio's teaching career, the content and development of his courses, his teaching methodology, his transition from professor of Legal philosophy to professor of Political philosophy, and about his particular admiration and respect for the classics. In this way, as an act of farewell to his teacher, the author presents his vision of both the human and the teaching facets of Norberto Bobbio through anecdotes, words, gestures and aspects of the philosopher's personality. 


\section{La última clase}

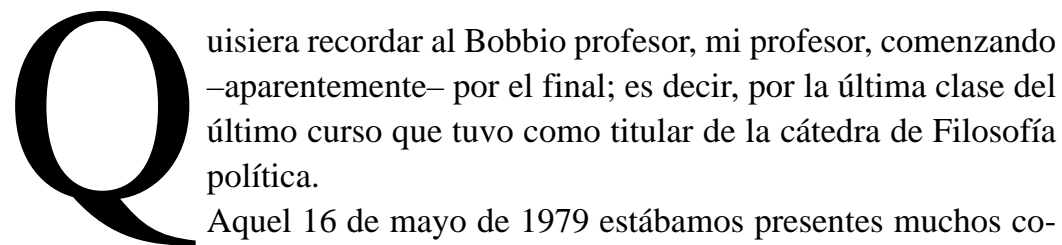
legas, en el aula 14 del primer piso del Palacio Nuevo. Bobbio empezó con estas palabras: «Pienso que no soy demasiado perspicaz si noto que en esta aula hay personas que no son estudiantes. Algunos de ellos han sido mis estudiantes; incluso, veo uno que debo haber reprendido durante una clase $\mathrm{y}$, algunas veces, cuando lo encuentro, me lo reprocha. [...] Debo decir que la presencia de estos "estudiantes no-estudiantes", no obstante haber pasado cuarenta y cuatro años de cuando comencé a enseñar, me pone un poco incómodo. Pero no tan incómodo como la primera vez que di una clase, en diciembre de 1935, en la Universidad de Camerino». Bobbio recordó que también en su primera clase estuvieron presentes muchos colegas de entonces, más bien, eran más numerosos que los estudiantes. Revivió aquel comienzo suyo con tono bromista, luego continuó en el hilo de la ironía: «Algún día he dicho a los estudiantes que quería evitar el rito de la última clase, no haciéndola. Más bien, habría quedado muy bien si ayer, que debió ser un día de huelga, luego anulada, no hubiera podido dar clase: porque hoy habría podido hacer... la penúltima». En este punto, propuso reemplazar la última clase con un diálogo: una discusión sobre los problemas tratados en el curso, que fue dedicada, en la primera parte, al cambio político como tema recurrente en la historia del pensamiento a partir de Aristóteles; y en la última parte, a la construcción de una teoría de la revolución, comparando los análisis y las reflexiones de los modernos sobre el fenómeno revolucionario, a partir de la Revolución francesa. En la discusión participaron, además de algunos estudiantes, muchos de los colegas concurrentes (puedo probarlo: el curso fue completamente registrado y luego trascrito por un grupo de alumnos).

Inició el diálogo. Bobbio contestó en su momento a las observaciones y también a las objeciones que le fueron planteadas, en el modo que le era usual: o sea, reconstruyendo ante todo el sentido de cada intervención, casi repitiéndola con otras palabras, traduciéndola, por así decirlo, en su propio lenguaje, como para apoderarse de las razones del interlocutor, como para ver si pudiera hacerlas suyas, antes de valorarlas críticamente y eventualmente señalarle los límites o contraponerle otras razones. Era la misma actitud, el mismo método, que siempre ha adoptado con todos sus interlocutores, incluidos los interlocutores puramente ideales, o sea, los autores comentados durante sus cursos. 
Aquella última “clase no-clase” finalizó con un obsequio floral y un aplauso, durante el cual Bobbio imitó el gesto de los ciclistas que han llegado primeros a la meta.

\section{Los seminarios de los años sucesivos y el post-último curso}

Pero aquella última clase no sólo no fue una verdadera clase: sino que tampoco fue la última. Como profesor sin cargo, Bobbio tuvo cuatro seminarios ligados al curso de Filosofía política, uno por cada año académico, excepto el año 1981/82. En realidad, al menos tres (de cuatro), no fueron propiamente seminarios sino breves cursos de clases, dictados los miércoles, de enero a abril. En el año 1979/80 eligió el tema «Hobbes y la libertad», y lo desarrolló con el estilo y las técnicas predilectas de la filosofía analítica: recuerdo, entre otras, una clase de lógica deóntica sobre la tríada conceptual «permitido-obligatorio-prohibido». En los años 1982/83 y 1983/84 dedicó los seminarios a la teoría de la democracia, focalizando la atención, en el primero, sobre la relación entre democracia, liberalismo y socialismo, a partir del análisis de los varios sentidos de los conceptos de igualdad y de libertad; y, en el segundo, sobre las «transformaciones de la democracia», a partir de una comparación entre dos autores que, en diferentes tiempos, habían adoptado esta mismo expresión como título para un libro, interpretándola en modos opuestos: Vilfredo Pareto y Johannes Agnoli.

En el año 1981/82 no tuvo el seminario porque había aceptado la suplencia de la enseñanza de Ciencia de la política y permaneció en ella, en tanto el titular estaba de licencia. Entonces, tampoco el último curso oficial fue realmente el último. El post-último curso fue titulado «El gobierno. Historia y teoría». Ya que no me consta que desde entonces haya sido recordado, creo hacer algo útil reconstruyendo brevemente su desarrollo. Después de una clase introductoria sobre el lugar y el rol de la noción de gobierno en el ámbito de los conceptos fundamentales de la política, dedicó algunas otras clases al análisis de las metáforas clásicas del gobernador — el timonel, el pastor, el tejedor - y a su suerte en la historia. Luego, pasó a reconstruir en las líneas esenciales las teorías clásicas y modernas de las formas de gobierno, asumiendo como hilo conductor la distinción entre formas monocráticas y colegiadas, y reservando particular atención a la figura del gobierno mixto y a sus diferentes interpretaciones. En este punto planteó el problema de la contraposición entre buen gobierno y mal gobierno y de los diferentes criterios que se pueden adoptar para distinguirlos; subrayó la importancia de la distinción entre gobierno de las leyes y gobierno de los hombres y la predominante identificación en la historia de varias formas de gobierno "del hombre”, es decir monocráticas, con formas de mal gobierno: tiranía, despotismo, dictadura, cesarismo, bonapartismo. Después de una comparación analítica entre estas formas, apoyada 
en los textos clásicos, desarrolló algunas consideraciones sobre la figura del jefe carismático. Dedicó la parte conclusiva del curso a la alternativa entre gobierno mínimo y gobierno máximo y las últimas clases al análisis crítico del concepto de gobernabilidad y sus posibles decadencias.

En cada cambio de tema, al principio de cada nuevo capítulo del curso, Bobbio retomaba desde el inicio el análisis del pensamiento antiguo y moderno recorriendo, por así decirlo, la historia de la cultura occidental en toda su amplitud, y tomando categorías y modelos conceptuales no sólo de los grandes filósofos, de las grandes obras políticas y jurídicas, sino también de textos historiográficos, literarios, hasta poéticos. He deshojado rápidamente mis notas personales relativas a este curso sobre el concepto de gobierno y he contado más de un centenar de autores y textos citados, comentados, discutidos en la clase: de Solón y Píndaro, por las nociones de eunomia y nomos basileus, hasta Robert Nozick, por la teoría neoliberal del Estado mínimo, contenida en un libro del que apenas había salido la traducción italiana. En el curso sobre el cambio político, son muchos más los autores en los cuales se detuvo Bobbio. Hago notar que he recordado dos cursos inéditos, y que no son los únicos en permanecer como tales. Quiero subrayar que solamente en parte, quizás en una parte no preponderante, la riqueza teórica, la cantidad de pensamientos elaborada por Bobbio en el círculo virtuoso entre búsqueda y enseñanza ha confluido, también, en los escritos que componen su amplísima bibliografía. Las notas que preparaba para las clases, para cada clase, contenidas en el archivo confiado por él al Centro Gobetti, serán ciertamente una fuente inagotable de hallazgos.

\section{La lección de los clásicos}

Considerados en su totalidad, los cursos de Bobbio se pueden representar como una especie de diálogo abierto y de continua comparación con innumerables voces de la cultura de cada tiempo, siempre reconstruidas con sentido histórico y rigor filológico, pero oídas, repensadas y evaluadas por aquellos que pueden decirnos o sugerirnos, por analogía y por diferencia, no sólo sobre su mundo sino también sobre el nuestro.

Francis Bacon distinguía en forma alegórica tres figuras de estudiosos: las hormigas, capaces de acumular ingentes cantidades de materiales retiradas del entorno circunstante; las arañas, que secretan por su cuenta el propio hilo de modo endógeno; y, las abejas, que elaboran, transforman y dan sabor a cuanto van recogiendo. Para bromear con la alegoría baconiana podríamos decir que Bobbio ha sido una abeja particularmente laboriosa y siempre curiosa de explorar nuevos campos. Pero, naturalmente, a menudo volvía con gusto sobre ciertas flores preferidas, extrayendo cada vez nuevo alimento: me refiero a los clásicos, identificados por él sobre todo como 
aquellos autores que han «construido teorías-modelo de las que uno se sirve continuamente para comprender la realidad, incluso una realidad distinta de aquella de la cual la[s] derivaron y a la que se la[s] aplicaron, y se han convertido en el curso de los años en verdaderas categorías mentales». En el Prólogo a las primeras notas de Filosofía política, del año académico 1972/73, escribía: «Si hubiera querido dar a mis apuntes un título académico, con gusto los habría titulado La lección de los clásicos». Quiero añadir una anécdota (verdadera y documentable). Concluyendo la segunda lección del último curso, aquel sobre el cambio político, Bobbio se dirigió a los estudiantes de este modo: «Ayer, alguien me ha preguntado si mi curso era “de actualidad”, y yo he respondido con un gesto. ¡Por qué esta idea de que todo debe ser de actualidad...! Sí, está bien, la actualidad... pero démonos cuenta que no hemos nacido ayer, que el mundo no ha nacido ayer, que a lo más somos enanos, pequeños enanos -según la famosa metáfora acuñada en el Seicento- sobre los hombros de los gigantes del pasado. Pero para subir sobre sus hombros necesitamos hacer un cierto trabajo, no es una cosa tan simple. Por esto, empezamos por Aristóteles, empezamos por Platón».

En uno de los escritos autobiográficos se encuentra un pasaje que corrige y reformula la metáfora de los enanos y de los gigantes, y que ilustra mejor la actitud de Bobbio con respecto a los clásicos: «Mi respeto por los clásicos ha llegado al punto de no haber osado nunca, por retomar la conocida imagen, ponerme sobre sus hombros, enano sobre los hombros de los gigantes, pero más alto únicamente por estar agachado sobre sus hombros. Siempre he tenido la sensación que, si lo hubiera hecho, uno de ellos hubiera tenido el derecho de decirme, algo enfadado: "Hazme el favor, baja y ocupa tu lugar, que es a mis pies”. Me viene a la mente otra metáfora, suscitada en mí por la afición a las muchas excursiones en la montaña hechas en compañía de mi esposa, primero con mis hijos y ahora con mis nietos: aquella del niño en el saco a la espalda. El adulto va delante y le señala el camino. El cansancio de la subida es todo suyo. El niño en el saco se deja llevar y, aunque a veces pueda dormirse, llega igualmente a la meta». En mi opinión, esta última metáfora habría que descifrarla con atención. El clásico “señala el camino”, un camino inaccesible, “en subida”, atravesado por difíciles problemas de comprensión y de interpretación de la realidad: hace falta seguirlo —un poco como decimos que se "sigue” una clase_ casi “dejándose llevar”, casi adormeciendo el impulso de desviarse del camino; el espíritu crítico debe volver a despertarse del todo en la meta, para valorar dónde has sido conducido, y si desde aquella meta puedes ver más, entender mejor.

El primer curso de filosofía política “seguía”, esto es, reconstruía y exploraba, cinco caminos, es decir, cinco recorridos teóricos, siempre comparando los sucesivos con los precedentes: los de Hobbes, Locke, Rousseau, Hegel y 
Marx. En la formulación y en la estructura, era muy similar al último curso de Filosofía del derecho, llevado a cabo en el año académico 1971/72, en el cual Bobbio había explorado, después de Marx, también los caminos de Max Weber y de Kelsen. Aquello que en el curso jurídico fue el objetivo principal, la reconstrucción de diferentes concepciones del Estado hasta la reducción kelseniana del Estado al ordenamiento jurídico, en el primer curso de Filosofía política se convertía, con una variación de énfasis, en la comparación de modelos conceptuales correspondientes a configuraciones alternativas de la gran dicotomía entre sociedad y Estado. Además, Bobbio se había ocupado de estos mismos y de otros clásicos en la mayor parte de los cursos jurídicos anteriores. Habiendo seguido unos y otros, puedo añadir, que en los últimos cursos de Filosofía del derecho era frecuente el recurso a los autores y modelos conceptuales no jurídicos en sentido estricto sino, más ampliamente, políticos; mientras en los cursos de Filosofía política era insistente la remisión a textos, a traducciones y sobre todo a modos lingüísticos más propiamente jurídicos. También por esto, en la última carta dirigida al decano y a los colegas de esta Facultad, con ocasión del seminario que organizamos por sus noventa años, Bobbio podía recordar el paso de una enseñanza a otra con las siguientes palabras: «Este paso fue para mí no solo indoloro sino, incluso, grato. Indoloro, porque, alternando cursos de argumento teórico con cursos de argumento histórico, había tenido frecuentes ocasiones en la enseñanza de la filosofía jurídica de afrontar tradicionales argumentos de filosofía política de Aristóteles, Locke, Kant, y un año el tema de la paz y de la guerra. Grato, porque la nueva enseñanza me permitía afrontar, como solamente la preparación de un curso puede hacer, temas de los cuales ya me había ocupado en los escritos precedentes, como la teoría de las formas de gobierno y el concepto de revolución. Dos cursos que recuerdo con particular gusto [...]. Siempre he considerado la esfera del derecho y la de la política, por usar una metáfora que me es familiar, como dos caras de la misma medalla. El mundo de las reglas y el mundo del poder. El poder que crea las reglas, las reglas que transforman el poder de hecho en un poder de derecho».

\section{4. ¿Filosofía o teoría?}

Quisiera añadir unas pocas palabras sobre el modo en el cual Bobbio concebía la naturaleza de las dos disciplinas y, en consecuencia, interpretaba la tarea de enseñarlas. A pesar de haber afirmado en un escrito de 1984, o sea en tiempos que no se remontaba a sus inicios como estudioso, de considerarse “perteneciente a la familia de los filósofos”, Bobbio siempre ha hecho un uso prudente de la palabra "filosofía”, al menos desde finales de los años cuarenta, es decir desde la época que coincide con su retorno a la Universidad de Turín. Sensible a las razones de la ciencia, era propenso a adoptar la fórmula 
comtiana de la "filosofía positiva”, aunque no a reconocerse completamente en ella, bien consciente de que esta fórmula le parecía a su propio inventor como inadecuada, una especie de oximorón al cual se podía recurrir faute de mieux. Su mal disimulada desconfianza por la palabra se habría originado, probablemente, en la abierta hostilidad contra "la última exaltación metafísica” de la cual había sido invadida nuestra cultura, el idealismo gentiliano, que casi llegó a identificarse y a ser identificado con "la” filosofía, y que Bobbio consideraba expresión emblemática de una pertinaz "ideología italiana”.

Estas actitudes y estos juicios de Bobbio se reflejaban no sólo en el modo de concebir sino hasta de nombrar las materias de su enseñanza. Ha permanecido célebre su contraposición entre "la filosofía del derecho de los filósofos y la filosofía del derecho de los juristas”, irónicamente vuelta contra los primeros. Menos conocida, quizás, sea su toma de distancia del modo de interpretar la filosofía política, convertida en hegemónica en las últimas décadas, como filosofía normativa de la justicia. En todo caso, para definir su enseñanza, prefirió el término “teoría” a la palabra filosofía. Lo afirmó con claridad, incluso con algún esfuerzo, en una conferencia de 1980, respondiendo a la pregunta “¿Qué hacen hoy los filósofos?”: «...he enseñado por muchos años dos materias filosóficas, la filosofía del derecho y la filosofía de la política, pero tanto una como otra, tal como yo las entiendo tienen muy poco que ver, a mi juicio, con la Filosofía, con mayúscula. [...] La mayor parte de los apuntes que han estudiado los alumnos no los he titulado Filosofía de..., sino siempre Teoría general del derecho, Teoría general de la política, Teoría de las formas de gobierno, etcétera».

Ahora bien: ya que consideraba el derecho y la política como dos caras de la misma medalla, del mismo modo concebía la filosofía del derecho y la filosofía política, interpretadas ambas en la forma de la "teoría general”, como disciplinas afines, contiguas y complementarias. En una reciente ocasión, había sintetizado su pensamiento con estas palabras: «esto que las dos teorías tienen en común en mis escritos [...] no solamente es el fin, exclusivamente cognoscitivo (no propositivo), sino también el modo de proceder para obtenerlo. Es el procedimiento [de la] “reconstrucción”, a través del análisis lingüístico nunca separado de las referencias históricas a los autores clásicos, de las categorías fundamentales, que permiten delimitar al exterior y ordenar al interior las dos áreas, aquella jurídica y aquella política, y [de establecer] sus relaciones recíprocas».

En realidad, a Bobbio le corresponde como a poquísimos otros el nombre de filósofo en el sentido más amplio: en el significado kantiano del que profesa el uso público de la razón. Pero para la enseñanza prefería mantenerse dentro de los límites de la "teoría general” entendida como análisis avalutativo de los conceptos fundamentales del derecho y de la política. 
Michelangelo Bovero

\section{No es un adiós}

Permitidme, para concluir, revivir la imagen de Bobbio durante las clases. Casi siempre caminaba de un sitio para otro, sin cesar, entre el pupitre y la pizarra deteniéndose de vez en cuando para trazar con la tiza un esquema conceptual. Comúnmente, permanecía sentado solamente cuando leía y comentaba pasajes de los clásicos. A menudo, como queriendo subrayar una frase o un pensamiento, golpeteaba rítmicamente con el dedo sobre la tabla de la mesa, suscitando inquietud en los estudiantes que habían acumulado las grabadoras sobre el pupitre. Recuerdo raras ocasiones en las cuales leía de pie, una página significativa, ligeramente inclinado hacia adelante. Después del asesinato de Moro recalcó palabra por palabra, sin énfasis, lentamente, el paso de Max Weber sobre la noche polar: ese que concluye con la exhortación «No importa, continuamos». Durante la última clase, aquel 16 de mayo de 1979, quiso recordar otra célebre afirmación de Weber, quizás la que le he oído repetir más veces: «La cátedra no es para los profetas ni para los demagogos». No sólo estas palabras, sino su tono de voz, siguen acompañándome.

A la página de los recuerdos se destinan los verbos en pasado. Una página que, por cuanto me concierne, pienso reabrir en público solamente una vez más, para luego guardarla en el secreto de la conciencia y releerla alguna otra vez junto a los amigos más cercanos. De las páginas acerca de los estudios sobre el pensamiento de Bobbio, ya de por sí numerosas, debemos pasar al presente histórico: «Bobbio dice ... Bobbio sostiene...». Sin embargo, en los recuerdos, me resulta difícil usar el perfecto. Tiendo a rechazarlo. Más espontáneo me viene el imperfecto, un tiempo inconcluso, que en cierto modo, de hecho, continúa: «Bobbio decía ... decía siempre...».

No logro decirle adiós. No podría. Estoy seguro que no podré. He releído, en estos días, el retrato de Leone Ginzburg trazado por Bobbio mucho tiempo después de la desaparición del amigo. En algunas de las palabras finales me ha parecido reconocerme, si parva licet, a mí mismo en el futuro, si me será dado, y aquel sentido íntimo de cercanía que seguiré teniendo con mi profesor: «el timbre de su voz, su mirada, su modo de hablar y de reír han quedado vivos en mi memoria, como si ayer mismo lo hubiera saludado por última vez. Si lo traigo a la mente, me sorprendo de sentirlo tan cercano, tan presente, tan junto a mí, dentro de mí, como si se hubiera convertido en parte de mí mismo».

(Trad. de Félix Morales Luna)

\section{DOXA 28 (2005)}

